Journal for ImmunoTherapy of Cancer

\title{
Pembrolizumab-induced cytokine release syndrome in a patient with metastatic lung adenocarcinoma: a case report
}

\author{
Paul Sackstein, ${ }^{1}$ Jacob Zaemes, ${ }^{1}$ Chul Kim (D) ${ }^{2}$
}

To cite: Sackstein P, Zaemes J, Kim C. Pembrolizumab-induced cytokine release syndrome in a patient with metastatic lung adenocarcinoma: a case report. Journal for ImmunoTherapy of Cancer 2021;9:e002855. doi:10.1136/jitc-2021-002855

- Additional supplemental material is published online only. To view, please visit the journal online (http://dx.doi.org/10. 1136/jitc-2021-002855).

Accepted 27 May 2021

\section{ABSTRACT}

Cytokine release syndrome (CRS) is a well-described immune-related adverse event following chimeric antigen receptor T-cell therapy, but has rarely been reported following anti-programmed death ligand-1 therapy. We report the case of a 55-year-old man with metastatic lung adenocarcinoma who presented with fever, chills and hypotension. Initial labs were notable for highly elevated serum ferritin levels and mildly elevated triglyceride levels. He was ultimately diagnosed with pembrolizumab-induced CRS complicated by multiorgan failure. The patient was treated with steroids and tocilizumab with normalization of inflammatory markers and resolution of renal failure. This case not only highlights the importance of considering CRS in patients who have developed multiorgan failure after immune checkpoint inhibitor therapy, but also demonstrates clinical similarities between CRS and other hyperinflammatory states such as hemophagocytic lymphohistiocytosis.

\section{INTRODUCTION}

Immune checkpoint inhibitor (ICI) therapy has revolutionized the treatment of nonsmall cell lung cancer (NSCLC), resulting in improved overall survival in both the first-line and second-line settings in advanced stage disease. ${ }^{1}$ While efficacious, such treatments are associated with a variety of immune-related adverse events (IRAEs). In patients receiving chimeric antigen receptor (CAR) T-cell therapy, cytokine release syndrome (CRS) and hemophagocytic lymphohistiocytosis (HLH) are well-described treatment-related complications. ${ }^{23}$ In rare instances, pembrolizumab, a programmed death ligand-1 (PD-L1) inhibitor, has also been reported to cause CRS and HLH in patients with advanced NSCLC. ${ }^{4}$ While CRS and HLH are distinct clinical entities, it can be challenging to differentiate the two as ICI therapy-induced HLH has also been recently described in the literature. ${ }^{6}$ In this report, we present a challenging case of pembrolizumab-induced CRS successfully treated with steroids and tocilizumab.

\section{CASE PRESENTATION}

A never-smoker 55-year-old man with a history of hyperlipidemia presented with hemoptysis accompanied by right shoulder pain and numbness. CT revealed a $5.6 \mathrm{~cm}$ right upper lobe spiculated mass with satellite nodularity concerning for lymphangitic spread. Positron emission tomography showed multifocal involvement of the right lung, mediastinal lymphadenopathy and widespread osseous metastases. MRI of the brain demonstrated multiple intracranial metastases. He underwent bronchoscopy and was diagnosed with metastatic lung adenocarcinoma. Tumor PD-L1 expression was $>50 \%$ by immunohistochemistry. Molecular testing demonstrated an EGFR exon 20 mutation (H773_V774insAH), PMS2 K413E, KMT2A Q1033P, MET K1262R, MET E719D and mutations in TP53. He received two cycles of pembrolizumab, pemetrexed and carboplatin. He was premedicated with intravenous dexamethasone $12 \mathrm{mg}$ on day 1 of cycles 1 and 2. For cycle 3, he received pembrolizumab without chemotherapy to allow for stereotactic radiosurgery (SRS) for brain lesions. The patient's cough and shoulder pain improved with treatment. Restaging MRI of the brain and CT of the chest/abdomen/pelvis after cycle 3 demonstrated reduction in size of the brain and lung lesions. Following the brain SRS, he completed a 20-day dexamethasone taper.

Approximately 19 days after cycle 3, he presented to the emergency department with fever and chills. On admission, vital signs were notable for fever $102.0^{\circ} \mathrm{F}$, blood pressure $64 / 44 \mathrm{~mm} \mathrm{Hg}$, heart rate 87 beats per minute and respiratory rate 35 breaths per minute with an oxygen saturation of $92 \%$ on room air. Physical examination was notable for lethargy and increased work of breathing. Initial labs demonstrated highly elevated inflammatory markers (ferritin, erythrocyte 
Table 1 Laboratory values on admission, after the first dose of tocilizumab and at the time of hospital discharge

\section{Laboratory tests}

\begin{tabular}{|c|c|c|c|c|c|}
\hline $\begin{array}{l}\text { Inflammatory } \\
\text { markers }\end{array}$ & Reference values & On admission & $\begin{array}{l}\text { Tocilizumab dose } \\
\text { \#1 (day 9) }\end{array}$ & $\begin{array}{l}\text { Tocilizumab dose } \\
\text { \#2 (day 16) }\end{array}$ & $\begin{array}{l}\text { On hospital } \\
\text { discharge (day } \\
27 \text { ) }\end{array}$ \\
\hline LDH (U/L) & $87-241$ & 3465 & 1515 & 1079 & 750 \\
\hline $\mathrm{ESR}(\mathrm{mm} / \mathrm{h})$ & $0-16$ & 85 & 30 & 2 & NA \\
\hline IL-6 (pg/mL) & $\leq 5$ & 75 & NA & NA & NA \\
\hline \multicolumn{6}{|l|}{ Liver function tests } \\
\hline AST (U/L) & $3-34$ & 536 & 119 & 120 & 75 \\
\hline ALT (U/L) & $15-41$ & 384 & 151 & 132 & 141 \\
\hline ANC (x10^9/L) & $>1500$ & 658 & 7000 & 11,100 & 3063 \\
\hline ALC (x10^9/L) & $600-4900$ & 579 & 600 & 700 & 1000 \\
\hline Hemoglobin (g/L) & $125-165$ & 114 & 69 & 70 & 82 \\
\hline $\begin{array}{l}\text { Platelet count } \\
(x 10 \wedge 9 / L)\end{array}$ & $145-400$ & 191 & 254 & 113 & 119 \\
\hline \multicolumn{6}{|c|}{ Basic metabolic panel } \\
\hline Creatinine (mg/dL) & $0.66-1.50$ & 1.9 & 8.4 & 3.4 & 1.1 \\
\hline
\end{tabular}

ALC, absolute lymphocyte count; ALP, alkaline phosphatase; ALT, alanine serum aminotransferase; ANC, absolute neutrophil count; AST, aspartate serum aminotransferase; CRP, C reactive protein; ESR, erythrocyte sedimentation rate; LDH, lactate dehydrogenase; sIL-2R, soluble IL-2 receptor; WBC, white blood cell.

sedimentation rate and $\mathrm{C}$ reactive protein) as well as elevated lactate dehydrogenase (LDH), serum aminotransferases, acute kidney injury, absolute lymphocytopenia with normal hemoglobin and platelet count, and moderate neutropenia with an absolute neutrophil count of 658 cells $/ \mu \mathrm{L}$ (table 1 ). X-ray of the chest demonstrated known right apical mass without new consolidation. $\mathrm{He}$ was admitted to the intensive care unit for vasopressor support, and received intravenous fluid resuscitation and empiric broad-spectrum antibiotics for possible bacterial infection.

The differential diagnoses included COVID-19 infection, tuberculosis, bacterial pneumonia, viral hepatitis, adrenal insufficiency and IRAEs, such as CRS and HLH. The patient tested negative for COVID-19 infection on four separate occasions during the hospitalization. Extensive tests for infectious disease including IFN-gamma release assay (T-SPOT), hepatitis A virus IgM and IgG serologies, hepatitis B surface antigen, hepatitis B surface antibody, Epstein-Barr virus IgM and IgG serologies, Bordatella pertussis antigen, HIV, aspergillus antigen, urine histoplasma, and urine/sputum/blood cultures on multiple occasions were unrevealing. A respiratory viral panel was negative for influenza A/B, parainfluenza
1/2/3/4, adenovirus, coronavirus (not COVID-19), rhinovirus/enterovirus, herpes simplex virus (HSV)-1, HSV-2, human metapneumovirus, mycoplasma pneumoniae and respiratory syncytial virus. Serum cytomegalovirus PCR was $2902 \mathrm{IU} / \mathrm{mL}$, which was felt to represent viral shedding in the setting of acute illness rather than active infection. A morning cortisol level was normal at $19.6 \mu \mathrm{g} / \mathrm{dL}$. Soluble IL-2R (sIL-2R) and interleukin 6 (IL-6) were found to be significantly elevated (table 1). A bone marrow biopsy could not be obtained due to his critical condition. In light of the negative laboratory workup for infectious disease and other etiologies including adrenal insufficiency, the patient was diagnosed with pembrolizumab-induced CRS and treated with intravenous methylprednisolone $1000 \mathrm{mg}$ once a day for 5 days, followed by methylprednisolone $2 \mathrm{mg} / \mathrm{kg}$ daily. Shortly after initiation of steroid treatment, the patient defervesced and was successfully weaned from vasopressors. The patient's serum inflammatory markers also demonstrated marked improvement (figure 1). Since the patient previously lived in Cambodia, he was empirically treated for possible chronic strongyloidiasis with a 2-day course of ivermectin. He developed anuric acute kidney injury and started hemodialysis on day 6 , which he received till 

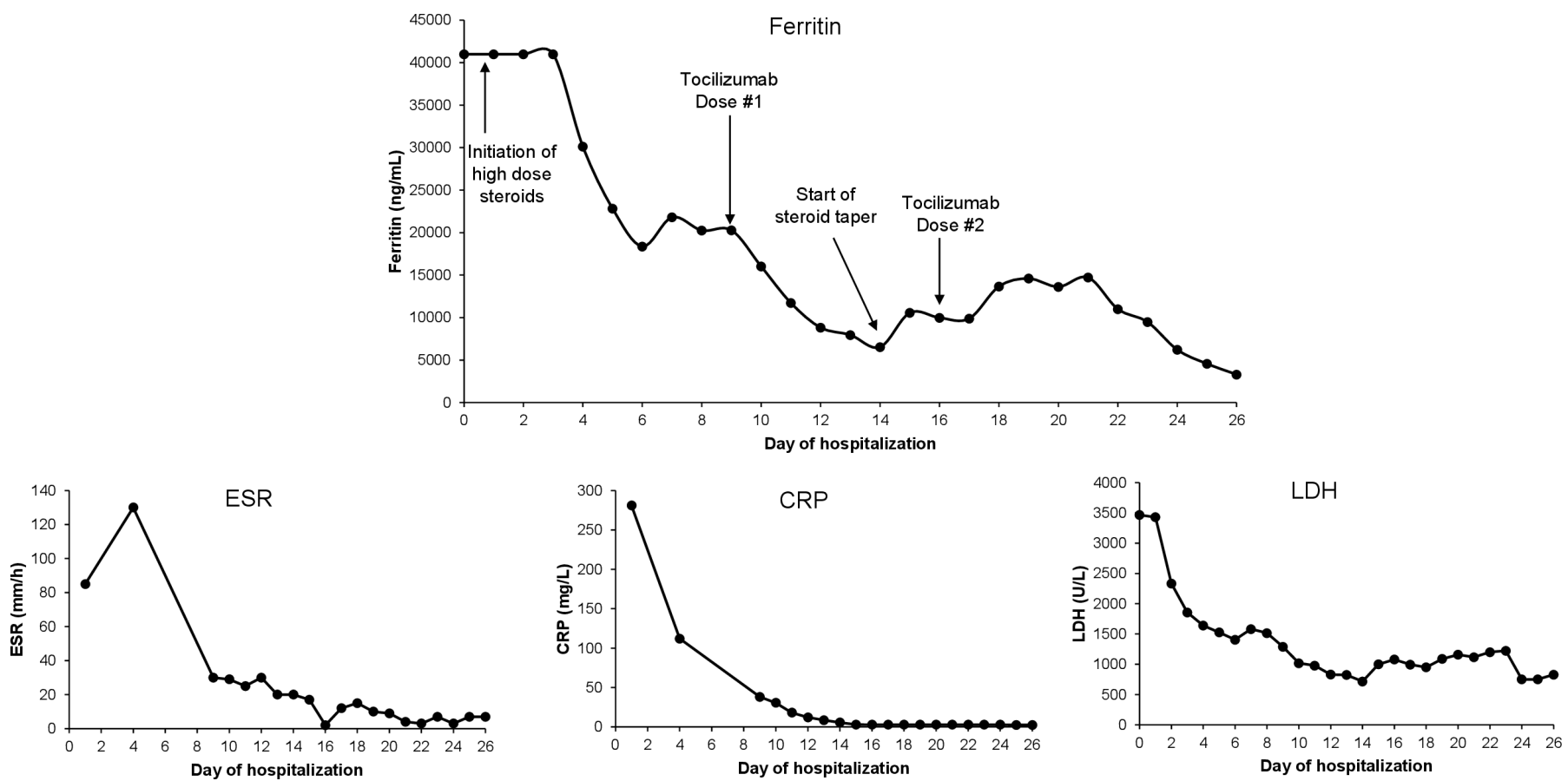

Figure 1 The downward trend of various inflammatory markers including ferritin, erythrocyte sedimentation rate (ESR), $C$ reactive protein $(\mathrm{CRP})$ and lactate dehydrogenase $(\mathrm{LDH})$ during the hospitalization. The graph of serum ferritin over time includes arrows denoting time points for the initiation of steroids, the beginning of the steroid taper and the two doses of tocilizumab administered.

day 14 . The patient developed rapidly worsening encephalopathy with somnolence and inability to speak on day 9 and serum ferritin had plateaued around $20,000 \mathrm{ng} /$ $\mathrm{mL}$ (figure 1). An electroencephalogram showed findings consistent with non-specific global encephalopathy and did not reveal any evidence of clinical or subclinical seizures. CT of the brain showed unchanged metastatic disease. In light of the patient's clinical deterioration, he was treated with intravenous tocilizumab $8 \mathrm{mg} / \mathrm{kg}$ on day 9 with increase in methylprednisolone to $1000 \mathrm{mg}$ once a day for 5 days. Tocilizumab was given again on day 16 of admission given ongoing encephalopathy and uptrending of inflammatory markers (ferritin, LDH). With high-dose steroids and tocilizumab, the patient's serum ferritin as well as his encephalopathy and anuric renal failure improved. With improvement in symptoms and organ function and normal vital signs, he transitioned to oral dexamethasone, which was tapered off over 4 weeks. The patient was successfully discharged from the hospital 27 days after admission.

\section{DISCUSSION}

CRS classically presents with fever and, in severe cases, hypotension and multiorgan failure after receiving cancer immunotherapy. Recently, CRS has been described in the literature in patients with metastatic NSCLC treated with pembrolizumab. ${ }^{45}$ Our patient presented with high fever, hypotension and multiorgan failure, but also had markedly elevated ferritin and sIL-2R levels, raising concern for HLH. He had absolute lymphocytopenia, but no evidence of thrombocytopenia, anemia or organomegaly. He did not meet the HLH 2004 diagnostic criteria and his HScore was 145, denoting a 16\%-25\% probability of HLH. ${ }^{78}$ Taken together, his clinical presentation was most consistent with pembrolizumab-induced CRS rather than HLH, although the latter could not be definitively excluded in the absence of a bone marrow biopsy. The elevated ferritin was felt to correlate with the severity of CRS and normalized following treatment of the inflammatory syndrome.

Our patient initially received chemoimmunotherapy but was treated with pembrolizumab alone during cycle 3, as he received brain SRS. He was premedicated with dexamethasone during cycles 1 and 2 and completed a dexamethasone taper following brain SRS. Interestingly, the patient developed CRS at the completion of a prolonged dexamethasone taper whereas most cases of CAR T-cell therapy-related CRS develop within 2-3 days of treatment. ${ }^{2}$ We hypothesize that the use of dexamethasone may have contributed to the delayed onset of the CRS in our patient. Also, it is possible that ICI-related CRS may have distinct onset and manifestations compared with CAR T-cell therapy-related CRS.

Management of CRS includes treatment with high-dose steroids with the addition of tocilizumab, an IL-6 inhibitor, for severe cases. ${ }^{9}$ Three cases of pembrolizumabrelated CRS have been reported in the literature, all of which improved following administration of intravenous methylprednisolone. ${ }^{4510}$ Our case was clinically similar to other reported cases of pembrolizumab-induced CRS, 
but steroids alone were not sufficient to improve the patient's condition. To our knowledge, this is the first case of pembrolizumab-induced CRS successfully treated with steroids and tocilizumab. Whereas, most cases of severe CAR T-cell therapy-induced CRS usually improve within several days of initiation of immunosuppressive agents such as tocilizumab and high-dose corticosteroids, our patient required a prolonged course of steroids and multiple doses of tocilizumab due to persistent multiorgan failure and neurologic toxicity. The natural history, manifestations and management of ICI-related CRS need to be better understood with future studies. Lastly, we cannot exclude the possibility of CRS related to neoantigen release following radiation, which has recently been described in the literature. ${ }^{11}$

\section{CONCLUSION}

CRS and HLH are distinct hyperinflammatory states with often similar clinical presentations. Our case highlights that an elevated ferritin $>10,000 \mathrm{ng} / \mathrm{mL}$ may be insufficient to differentiate these conditions. Prompt recognition of CRS is critical as administration of steroids and tocilizumab can reverse multiorgan failure if administered early.

\section{Twitter Chul Kim @chulkimMD}

Contributors PS performed the literature review, constructed the table and figure, and wrote the initial draft. JZ and CK assisted in revision of the manuscript as well as the associated table and figure.

Funding The authors have not declared a specific grant for this research from any funding agency in the public, commercial or not-for-profit sectors.

Competing interests No, there are no competing interests.

Patient consent for publication Obtained from the patient's family daughter (power of attorney).

Ethics approval Obtained from the patient's family daughter (power of attorney).

Provenance and peer review Not commissioned; externally peer reviewed.

Supplemental material This content has been supplied by the author(s). It has not been vetted by BMJ Publishing Group Limited (BMJ) and may not have been peer-reviewed. Any opinions or recommendations discussed are solely those of the author(s) and are not endorsed by BMJ. BMJ disclaims all liability and responsibility arising from any reliance placed on the content. Where the content includes any translated material, BMJ does not warrant the accuracy and reliability of the translations (including but not limited to local regulations, clinical guidelines, terminology, drug names and drug dosages), and is not responsible for any error and/or omissions arising from translation and adaptation or otherwise.

Open access This is an open access article distributed in accordance with the Creative Commons Attribution Non Commercial (CC BY-NC 4.0) license, which permits others to distribute, remix, adapt, build upon this work non-commercially, and license their derivative works on different terms, provided the original work is properly cited, appropriate credit is given, any changes made indicated, and the use is non-commercial. See http://creativecommons.org/licenses/by-nc/4.0/.

\section{ORCID iD}

Chul Kim http://orcid.org/0000-0003-0191-8684

\section{REFERENCES}

1 Mok TSK, Wu Y-L, Kudaba I, et al. Pembrolizumab versus chemotherapy for previously untreated, PD-L1-expressing, locally advanced or metastatic non-small-cell lung cancer (KEYNOTE-042): a randomised, open-label, controlled, phase 3 trial. Lancet 2019;393:1819-30.

2 Davila ML, Riviere I, Wang X, et al. Efficacy and toxicity management of 19-28z CAR T cell therapy in B cell acute lymphoblastic leukemia. Sci Transl Med 2014;6:224ra225.

3 Sadaat M, Jang S. Hemophagocytic lymphohistiocytosis with immunotherapy: brief review and case report. J Immunother Cancer 2018;6:49.

4 Kogure $\mathrm{Y}$, Ishii $\mathrm{Y}$, Oki M. Cytokine release syndrome with pseudoprogression in a patient with advanced non-small cell lung cancer treated with pembrolizumab. J Thorac Oncol 2019;14:e55-7.

5 Rassy EE, Assi T, Rizkallah J, et al. Diffuse edema suggestive of cytokine release syndrome in a metastatic lung carcinoma patient treated with pembrolizumab. Immunotherapy 2017;9:309-11.

6 Honjo O, Kubo T, Sugaya F, et al. Severe cytokine release syndrome resulting in purpura fulminans despite successful response to nivolumab therapy in a patient with pleomorphic carcinoma of the lung: a case report. $J$ Immunother Cancer 2019;7:97.

7 Henter J-I, Horne A, Aricó M, et al. HLH-2004: diagnostic and therapeutic guidelines for hemophagocytic lymphohistiocytosis. Pediatr Blood Cancer 2007;48:124-31.

8 Fardet L, Galicier L, Lambotte O, et al. Development and validation of the HScore, a score for the diagnosis of reactive hemophagocytic syndrome. Arthritis Rheumatol 2014;66:2613-20.

9 Cobb DA, Lee DW. Cytokine release syndrome biology and management. Cancer J 2021;27:119-25.

10 Normand CV, Zender HO, Staehli DM, et al. Acute cytokine release syndrome after a first dose of pembrolizumab as second-line treatment for metastatic, programmed death-ligand 1-positive, nonsmall-cell lung cancer. J Oncol Pharm Pract 2020:107815522098081.

11 Barker CA, Kim SK, Budhu S, et al. Cytokine release syndrome after radiation therapy: case report and review of the literature. $J$ Immunother Cancer 2018;6:1. 